\title{
Variabilidad vegetativa intra e interespecífica de Astrocaryum chonta y A. javarense (Arecaceae) en Jenaro Herrera, Loreto, Perú
}

\author{
Intra and interspecific vegetative variability of Astrocaryum chonta and A. javarense (Arecaceae) \\ from Jenaro Herrera, Loreto, Peru
}

Miguel Machahua, Francis Kahn y Betty Millán

Museo de Historia Natural, Universidad Nacional Mayor de San Marcos, Av. Arenales 1256, Jesús María, Lima 11, Perú.

Email Miguel Machahua:m.machahua@gmail.com Email Francis Kahn: franciskahn@orange.fr Email Betty Millán: bmillans@gmail.com

Citación:

Machahua M., F. Kahn \& B. Millán. 2014. Variabilidad vegetativa intra e interespecífica de Astrocaryum chonta y $A$. javarense (Arecaceae) en Jenaro Herrera, Loreto, Perú. Revista peruana de biología 21(2): 139 - 144 (Octubre 2014). doi: http://dx.doi.org/10.15381/rpb.v21i2.9816

\begin{abstract}
Resumen
Los caracteres vegetativos cuantitativos de individuos adultos, juveniles II, juveniles I y plántulas de Astrocaryum chonta y Astrocaryum javarense de cuatro poblaciones de la zona de Jenaro Herrera fueron analizados para observar los patrones de variación vegetativa a nivel intra e interespecífico. Los datos biométricos fueron analizados mediante ANOVA ( $p<0.05$ y 0.01). A nivel intraespecífico el $37 \%$ y $64 \%$ de los caracteres vegetativos son significativamente diferentes en Astrocaryum chonta y Astrocaryum javarense respectivamente; mientras a nivel interespecífico el $81 \%$ de los caracteres vegetativos son significativamente diferentes.
\end{abstract}

Palabras clave: Palmeras Huicungo; variabilidad vegetativa; Amazonia peruana.

\section{Abstract}

Vegetative quantitative characters from adult, juvenile II, juvenile I and seedlings of Astrocaryum chonta and Astrocaryum javarense for four populations of Jenaro Herrera were analyzed in order to observe the patterns of vegetative variation at intra and interspecific level. The biometric data were analyzed using ANOVA ( $p<0.05$ and 0.01 ). At intraspecific level the $37 \%$ and $64 \%$ of vegetative characters are significantly different in Astrocaryum chonta and Astrocaryum javarense, while at interspecific level the $81 \%$ are significantly different.

Keywords: Huicungo palms; vegetative variability; Peruvian Amazon.
Fuentes de financiamiento:

El presente trabajo se realizó dentro del Convenio entre UNMSM/Universidad Nacional Mayor de San Marcos, Perú y el IRD/Instituto de Investigaciones para el Desarrollo, Francia; y fue financiado por el Proyecto FP7 PALMS N²12631.

Informacion sobre los autores:

Los autores declaran que partciparon en la elaboración del trabajo realizando: MM: muestreo, análisis de datos y redacción, FK: análisis y redacción y BM: análisis y redacción.

\section{Introducción}

El género Astrocaryum presenta 40 especies, se encuentran comúnmente en los ecosistemas tropicales de Sudamérica y los bosques tropicales de América Central. El género se divide en 3 subgéneros: Astrocaryum con 16 especies, Munbaca con 4 especies y Monogynanthus con 20 especies (Kahn 2008).

La sección Huicungo del subgénero Monogynanthus es la más diversificada en el Perú con 12 de sus 15 especies (Kahn 2008, Kahn \& Millán 2013). Todas las especies están adaptadas al sotobosque de los bosques tropicales, pero también se desarrollan muy bien en áreas abiertas donde incluso llegan a ser invasoras (Vormisto et al. 2004, Aponte et al. 2011, Kahn \& Millán 2013). Las especies presentan alta similitud vegetativa, siendo todas palmeras con tronco corto a mediano y con hojas largas (Balslev et al. 2011, Kahn \& Millán 2013). Estudios sobre variabilidad vegetativa a nivel intra e interespecífica en este grupo de palmeras son escasos; Aponte et al. (2011) reportaron variabilidad morfológica en Astrocaryum perangustatum que crece en bosques y pastizales en Pozuzo (Perú).

Astrocaryum chonta y A. javarense pertenecen a la sección Huicungo. Sus áreas de distribución geográfica son contiguas, Astrocaryum chonta se encuentra distribuida en

$\begin{array}{ll}\text { Presentado: } & \text { 25/02/2014 } \\ \text { Aceptado: } & 04 / 06 / 2014\end{array}$

Publicado online: $07 / 10 / 2014$

Journal home page: http://revistasinvestigacion.unmsm.edu.pe/index.php/rpb/index

(C) Los autores. Este artículo es publicado por la Revista Peruana de Biología de la Facultad de Ciencias Biológicas, Universidad Nacional Mayor de San Marcos. Este es un artículo de acceso abierto, distribuido bajo los términos de la Licencia Creative Commons Atribución-NoComercial-Compartirlgual 4.0 Internacional.(http://creativecommons.org/licenses/by-nc-sa/4.0/), que permite el uso no comercial, distribución y reproducción en cualquier medio, siempre que la obra original sea debidamente citadas. Para uso comercial, por favor póngase en contacto con editor.revperubiol@gmail.com. 


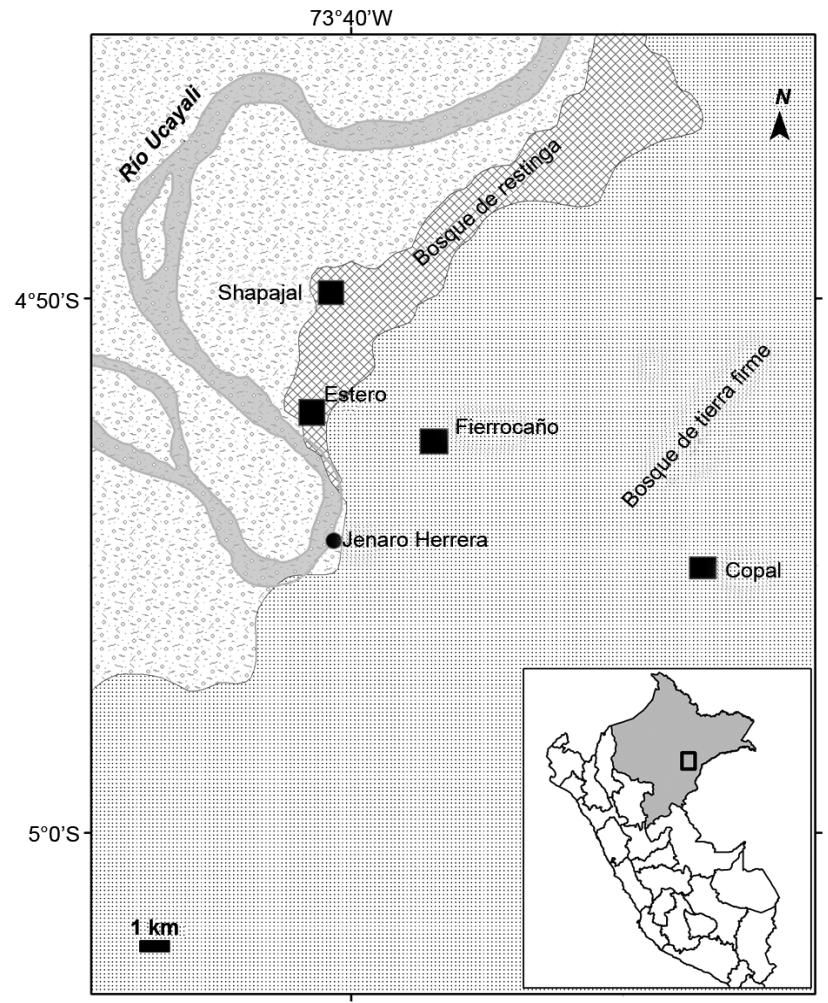

Figura 1. Área de estudio. Dos bosques de restinga (Estero y Shapajal) y dos bosques de altura (Copal y Fierrocaño) (adaptado del mapa Geológico del Perú-INGEMMET).

los bosques de restingas, mientras que $A$. javarense crece en los bosques de tierra firme (Kahn et al. 2011). Las dos especies se diferencian claramente por la morfología de sus partes florales y sus frutos (Kahn \& Millán, 2013). Sin embargo, distinguirlas sólo a partir de los caracteres vegetativos es mucho más difícil.

En el presente trabajo se realizó el análisis de la variabilidad de caracteres vegetativos a nivel intra e interespecífico de Astrocayum chonta y Astrocaryum javarense.

\section{Material y métodos}

\section{Especies estudiadas:}

Astrocaryum chonta Mart. .- Palmera monocaule. Tallo hasta $15 \mathrm{~m}$ de alto y $30 \mathrm{~cm}$ de diámetro. Hojas $8-16$, hasta 950 cm de largo; vaina y pecíolo hasta $230 \mathrm{~cm}$ de largo, fibroso, con aguijones aplanados, de color negro, hasta $20 \mathrm{~cm}$ de largo; raquis hasta $760 \mathrm{~cm}$ de largo, con tomento blanquecino y aguijones aplanados, de color negro, hasta $10 \mathrm{~cm}$ de largo; pinnas $89-130$ por lado, regularmente dispuestas en un mismo plano. Flores pistiladas con cáliz glabro a glabrescente. Fruto con epicarpo marrón, tomentoso, con setas suaves, flexuosas $2-3 \mathrm{~mm}$ de largo (Kahn \& Millán 2013).

Se distribuye en Bolivia y Perú (Kahn 2008); en el Perú se encuentra en las terrazas bajas de los ríos Amazonas-Ucayali, hasta el bajo Urubamba y Tambo, se encontró puntualmente en el bajo Marañón al Norte del río Ucayali en Loreto, así como en el valle del río Manu en Madre de Dios (Kahn et al. 2011).

Astrocaryum javarense Trail ex Drude .- Palmera monocaule. Subacaulescente o con tallo hasta de $2 \mathrm{~m}$ de altura, $15-18 \mathrm{~cm}$ de diámetro, de color marrón. Hojas 8 - 16; vaina y pecíolo hasta
$340 \mathrm{~cm}$ de largo, armada densamente con aguijones aplanados de color negro, hasta $30 \mathrm{~cm}$ de largo; raquis $270-570 \mathrm{~cm}$ de largo, lado abaxial con un indumento blanquecino y con setas de negras y aguijones hasta de $8 \mathrm{~cm}$ de largo, negros; pinnas 75 - 102 por lado, regularmente dispuestas en un mismo plano. Flores pistiladas con cáliz armado de aguijones flexuosos, de color amarillento, marrón o negro. Fruto con epicarpo flocoso, marrón, con aguijones flexuosos, de color marrón a negro, 4 - 8 mm de ancho (Kahn \& Millán 2013).

Se distribuye en Brasil y Perú (Lorenzi et al. 2010); en el Perú se encuentra en la región Sur del río Ucayali y Amazonas hasta el Sur de la cuenca del río Jauari (Kahn et al. 2011).

Áreas de estudio.- Comprende la localidad de Jenaro Herrera (45ㄴ $\mathrm{S}, 73^{\circ} 40^{\prime} \mathrm{W}$, altitud $125 \mathrm{~m}$ ), perteneciente al distrito de Jenaro Herrera, provincia de Requena, Región Loreto. El muestreo se realizó en bosques de restinga y bosques de tierra firme (Fig. 1).

Los bosques de restinga de Estero y Shapajal son terrenos de inundación temporal, se presentan en forma de terrazas, con fajas angostas, pequeñas y dispersas en forma paralela al cauce de los ríos (Rodríguez 1990); el suelo presenta una reacción básica ( $\mathrm{pH}$ de 6.4 a 7.8) con buena fertilidad (Hoag 1987, Rodríguez 1990). Los bosques de tierra firme de Fierrocaño y Copal son terrenos no inundables, se encuentran alejados de los cauces de los ríos (López Parodi y Freitas 1990); el suelo presenta una reacción acida ( $\mathrm{pH} 3.7$ a 5.3) con baja fertilidad (Escobedo et al. 1994). García (1975) reportó que los suelos de Copal presentan mayor fertilidad que los otros bosques de tierra firme de Jenaro Herrera.

Diseño de muestreo.- Para Astrocaryum chonta; en el bosque de restinga Estero, fueron muestreados 20 palmeras adultas, 12 juveniles II, 19 juveniles I y 58 plántulas; y en el bosque de restinga Shapajal: 20 adultos, 6 juveniles II, 18 juveniles I y 54 plántulas. Para Astrocaryum javarense se muestrearon en el bosque de tierra firme Fierrocaño: 20 adultos, 10 juveniles II, 15 juveniles I y 37 plántulas; y en el bosque de tierra firme Copal: 20 adultos, 12 juveniles II, 10 juveniles I y 59 plántulas.

Para el reconocimiento de los estadios de desarrollo se adoptaron los protocolos de Anthelme et al. (2010). El individuo tipo plántula se caracteriza por presentar hojas con el limbo entero y bífido, el individuo tipo juvenil I presenta hojas de 0.8 a $2 \mathrm{~m}$ de longitud con limbo pinnado irregularmente, el individuo juvenil II presenta hojas de 2 a $4 \mathrm{~m}$ de longitud con limbo pinnado regularmente, y el individuo adulto presenta hojas de más de 4 $\mathrm{m}$ de largo con limbo pinnado regularmente.

Parámetros biométricos.- Para los adultos, juveniles II y juveniles I se registraron datos cuantitativos de caracteres vegetativos: altura total de la palmera, largo del tronco, diámetro del tronco, número de hojas en la corona, número de pinnas (ambos lados), largo del vaina-pecíolo, largo del raquis, largo de la pinna distal, ancho de la pinna distal, largo de la pinna media, ancho de la pinna media, largo de la pinna proximal, ancho de la pinna proximal y largo de 5 aguijones más grandes de la vaina. Para los individuos adultos se registraron datos de 3 hojas, los juveniles II y I no se registraron datos del tronco por no presentar tallo. Para las plántulas se registraron el número de hojas, largo del pecíolo, largo del raquis y ancho de lámina. 
Análisis estadístico.- Los datos fueron analizados mediante la estadística descriptiva y el análisis de varianza ANOVA ( $\mathrm{p}<0.01$; 0.05) a nivel intra e interespecífico. Todos los análisis fueron realizados con el paquete estadístico SPSS Inc. (2008).

\section{Resultados}

Variabilidad vegetativa en Astrocaryum chonta (Tabla 1).(1) Seis caracteres son significativamente diferentes en adultos: largo del tronco, largo de la vaina-pecíolo, largo del raquis, ancho de la pinna proximal y largo de los aguijones. (2) Tres caracteres son significativamente diferentes en juveniles II: largo de la pinna media, largo de la pinna proximal y largo de los aguijones. (3)
Tres caracteres son significativamente diferentes en juveniles I: número de hojas, ancho de la pinna distal y largo de los aguijones. (4) Dos caracteres son significativamente diferentes en plántulas: largo del raquis y ancho de la lámina.

Variabilidad vegetativa en Astrocaryum javarense (Tabla 2).(1) Diez caracteres son significativamente diferentes en adultos: largo del tronco, número de hojas, número de pinnas, largo de la vaina-pecíolo, largo del raquis, largo de la pinna distal, largo de la pinna media, ancho de la pinna media, largo de la pinna proximal y largo de los aguijones. (2) Seis caracteres son significativamente diferentes en juveniles II: altura total, número de

Tabla 1. Variabilidad vegetativa en Astrocaryum chonta: adultos, juveniles II, juveniles I y plántulas en los bosques de restinga Estero y Shapajal $\left({ }^{* *} p<0.01 ;{ }^{*} p<0.05 ;-p>0.05\right)$.

\begin{tabular}{|c|c|c|c|c|c|}
\hline \multirow{2}{*}{ Adultos } & \multicolumn{2}{|r|}{ Estero } & \multicolumn{2}{|r|}{ Shapajal } & \multirow{2}{*}{$p$} \\
\hline & $\mathbf{N}$ & Media \pm DS (min-max) & $\mathbf{N}$ & Media \pm DS (min-max) & \\
\hline Altura total $(\mathrm{cm})$ & 20 & $942.0 \pm 218.2(700-1400)$ & 20 & $954.5 \pm 189.0(720-1420)$ & - \\
\hline Largo tronco $(\mathrm{cm})$ & 20 & $290.5 \pm 192.2(50-600)$ & 20 & $477.0 \pm 160.0(150-700)$ & $* *$ \\
\hline Diámetro tronco $(\mathrm{cm})$ & 20 & $18.8 \pm 5.5(11.9-27.7)$ & 20 & $16.0 \pm 4.0(9-26)$ & - \\
\hline Número de hojas & 20 & $10.1 \pm 2.1(7-15)$ & 20 & $11.3 \pm 2.2(8-15)$ & - \\
\hline Número de pinnas & 120 & $103.2 \pm 11.9(74-124)$ & 120 & $103.3 \pm 10.7(79-130)$ & - \\
\hline Largo vaina-pecíolo (cm) & 60 & $196 \pm 32.1(133-282)$ & 60 & $182.4 \pm 22.7(120-240)$ & ** \\
\hline Largo raquis (cm) & 60 & $547.9 \pm 85.3(385-700)$ & 60 & $507.8 \pm 77.9(350-660)$ & ** \\
\hline Largo pinna distal $(\mathrm{cm})$ & 60 & $49.1 \pm 11.5(31-87)$ & 60 & $53 \pm 14.4(29-89)$ & - \\
\hline Ancho pinna distal $(\mathrm{cm})$ & 60 & $2.4 \pm 0.7(1.3-5)$ & 60 & $2.6 \pm 0.6(1.5-5)$ & - \\
\hline Largo pinna media $(\mathrm{cm})$ & 60 & $115.5 \pm 17.8(88-155)$ & 60 & $120.3 \pm 16.4(88-160)$ & - \\
\hline Ancho pinna media $(\mathrm{cm})$ & 60 & $5.9 \pm 1.1(4-8)$ & 60 & $6.2 \pm 1.1(4-9)$ & - \\
\hline Largo pinna proximal (cm) & 60 & $70.6 \pm 19.9(38-126)$ & 60 & $88-3 \pm 20.7(56-132)$ & ** \\
\hline Ancho pinna proximal (cm) & 60 & $2.4 \pm 0.9(1-7.2)$ & 60 & $3.1 \pm 0.9(1.5-6)$ & ** \\
\hline Largo aguijones vaina $(\mathrm{cm})$ & 100 & $16.9 \pm 5.7(8-33.6)$ & 100 & $15.2 \pm 5.2(5-31.5)$ & * \\
\hline \multicolumn{6}{|l|}{ Juveniles II } \\
\hline Altura total $(\mathrm{cm})$ & 12 & $332.5 \pm 121.1(200-600)$ & 6 & $309.2 \pm 81.9(230-420)$ & - \\
\hline Número de hojas & 12 & $7.6 \pm 1.6(5-11)$ & 6 & $6.5 \pm 2.2(4-10)$ & - \\
\hline Número de pinnas & 24 & $58.2 \pm 16.0(30-83)$ & 12 & $52.8 \pm 26.8(27-89)$ & - \\
\hline Largo vaina-pecíolo (cm) & 12 & $157.9 \pm 59.0(94-290)$ & 6 & $123 \pm 26.9(92-160)$ & - \\
\hline Largo raquis $(\mathrm{cm})$ & 12 & $248.5 \pm 52.9(182-350)$ & 6 & $302.8 \pm 88.7(150-380)$ & - \\
\hline Largo pinna distal (cm) & 12 & $38.3 \pm 12.0(19-59)$ & 6 & $49.3 \pm 13.5(36-75)$ & - \\
\hline Ancho pinna distal (cm) & 12 & $2.4 \pm 0.8(1-4)$ & 6 & $3.0 \pm 1.3(2-5.5)$ & - \\
\hline Largo pinna media $(\mathrm{cm})$ & 12 & $65.3 \pm 15.4(49-92)$ & 6 & $92.7 \pm 11.9(76-105)$ & ** \\
\hline Ancho pinna media $(\mathrm{cm})$ & 12 & $3.1 \pm 1.0(2-5)$ & 6 & $3.5 \pm 0.9(2-4.5)$ & - \\
\hline Largo pinna proximal $(\mathrm{cm})$ & 12 & $49.6 \pm 21.1(8-86)$ & 6 & $106.2 \pm 17.2(85-134)$ & ** \\
\hline Ancho pinna proximal $(\mathrm{cm})$ & 12 & $2.7 \pm 1.9(1-7)$ & 6 & $3.4 \pm 1.8(2-7)$ & - \\
\hline Largo aguijones vaina $(\mathrm{cm})$ & 60 & $3.6 \pm 2.3(0-7)$ & 30 & $6.5 \pm 2.2(3.5-12)$ & ** \\
\hline \multicolumn{6}{|l|}{ Juveniles I } \\
\hline Altura total $(\mathrm{cm})$ & 19 & $175.0 \pm 85.4(80-400)$ & 18 & $144.8 \pm 50.7(70-230)$ & - \\
\hline Número de hojas & 19 & $5.4 \pm 1.5(4-8)$ & 18 & $6.7 \pm 1.3(5-9)$ & ** \\
\hline Número de pinnas & 38 & $10.8 \pm 6.1(3-24)$ & 36 & $9.8 \pm 7.1(3-37)$ & - \\
\hline Largo vaina-pecíolo (cm) & 19 & $98.5 \pm 31.3(60-152)$ & 18 & $81.4 \pm 25.4(34-125)$ & - \\
\hline Largo raquis $(\mathrm{cm})$ & 19 & $99.7 \pm 47.0(26-200)$ & 18 & $94.8 \pm 37.3(41-212)$ & - \\
\hline Largo pinna distal (cm) & 19 & $51.2 \pm 14.2(14-80)$ & 18 & $58.6 \pm 14.4(27-79)$ & - \\
\hline Ancho pinna distal $(\mathrm{cm})$ & 19 & $4.8 \pm 3.9(1.5-14)$ & 18 & $8.0 \pm 3.5(2-12)$ & * \\
\hline Largo pinna media $(\mathrm{cm})$ & 19 & $55.6 \pm 12.2(33-76)$ & 18 & $59.4 \pm 11.9(38-78)$ & - \\
\hline Ancho pinna media $(\mathrm{cm})$ & 19 & $2.8 \pm 2.3(1.5-11)$ & 18 & $2.7 \pm 1.5(1.5-6.5)$ & - \\
\hline Largo pinna proximal $(\mathrm{cm})$ & 19 & $57.1 \pm 14.5(35-80)$ & 18 & $64.5 \pm 10.6(47-85)$ & - \\
\hline Ancho pinna proximal $(\mathrm{cm})$ & 19 & $3.1 \pm 1.5(1-13)$ & 18 & $3.1 \pm 1.5(1.5-6)$ & - \\
\hline Largo aguijones vaina $(\mathrm{cm})$ & 95 & $2.8 \pm 3.0(0-10)$ & 90 & $4.3 \pm 3.5(0-19)$ & ** \\
\hline \multicolumn{6}{|l|}{ Plántulas } \\
\hline Número hojas & 58 & $3.7 \pm 1.4(1-7)$ & 54 & $3.8 \pm 1.5(2-7)$ & - \\
\hline Largo pecíolo $(\mathrm{cm})$ & 58 & $19.5 \pm 5.8(7-37)$ & 54 & $18.3 \pm 9.0(8-54)$ & - \\
\hline Largo raquis $(\mathrm{cm})$ & 58 & $19.0 \pm 6.0(7-34)$ & 54 & $15.4 \pm 4.1(7-26)$ & $* *$ \\
\hline Ancho lámina $(\mathrm{cm})$ & 58 & $5.8 \pm 2.2(2-13)$ & 54 & $7.1 \pm 2.4(3-13)$ & ** \\
\hline
\end{tabular}


Tabla 2. Variabilidad vegetativa en Astrocaryum javarense: adultos, juveniles II, juveniles I y plántulas en los bosques de tierra firme Fierrocaño y Copal $\left({ }^{* *} p<0.01 ;{ }^{*} p<0.05 ;-p>0.05\right)$.

\begin{tabular}{|c|c|c|c|c|c|}
\hline \multirow{2}{*}{ Adultos } & \multicolumn{2}{|r|}{ Fierrocaño } & \multicolumn{2}{|r|}{ Copal } & \multirow{2}{*}{$p$} \\
\hline & $\mathbf{N}$ & Media \pm DS (min-max) & $\mathbf{N}$ & Media \pm DS (min-max) & \\
\hline Altura total $(\mathrm{cm})$ & 20 & $488.0 \pm 77.1(350-700)$ & 20 & $539.7 \pm 96.9(450-890)$ & - \\
\hline Largo tronco $(\mathrm{cm})$ & 20 & $50.8 \pm 3.4(50-65)$ & 20 & $89.0 \pm 67.1(50-300)$ & * \\
\hline Diámetro tronco (cm) & 20 & $10.2 \pm 0.9(10-14)$ & 20 & $13.0 \pm 8.9(10-15.9)$ & - \\
\hline Número de hojas & 20 & $7.3 \pm 1.7(5-10)$ & 20 & $8.7 \pm 1.9(6-13)$ & * \\
\hline Número de pinnas & 120 & $87.9 \pm 11.5(65-120)$ & 120 & $100.6 \pm 14.5(68-130)$ & ** \\
\hline Largo vaina-pecíolo (cm) & 60 & $186.9 \pm 35.3(120-270)$ & 60 & $209.3 \pm 52.2(130-360)$ & * \\
\hline Largo raquis (cm) & 60 & $400.4 \pm 64.7(265-536)$ & 60 & $476.1 \pm 84.4(220-623)$ & ** \\
\hline Largo pinna distal $(\mathrm{cm})$ & 60 & $39.8 \pm 10.5(20-66)$ & 60 & $49.9 \pm 10.1(32-80)$ & $* *$ \\
\hline Ancho pinna distal (cm) & 60 & $2.0 \pm 0.4(1-3)$ & 60 & $2.1 \pm 0.5(1-3.5)$ & - \\
\hline Largo pinna media (cm) & 60 & $80.8 \pm 11.9(62-135)$ & 60 & $98.8 \pm 15.3(60-130)$ & $* *$ \\
\hline Ancho pinna media $(\mathrm{cm})$ & 60 & $3.6 \pm 0.7(2-5)$ & 60 & $4.4 \pm 1.0(1-7)$ & ** \\
\hline Largo pinna proximal $(\mathrm{cm})$ & 60 & $55.1 \pm 13.5(34-89)$ & 60 & $65.5 \pm 15.2(22-105)$ & ** \\
\hline Ancho pinna proximal $(\mathrm{cm})$ & 60 & $1.9 \pm 3.0(1-3.5)$ & 60 & $1.9 \pm 0.6(1-3)$ & - \\
\hline Largo aguijones vaina $(\mathrm{cm})$ & 100 & $9.3 \pm 3.0(4-19)$ & 100 & $17.3 \pm 11.7(2.5-45)$ & $* *$ \\
\hline \multicolumn{6}{|l|}{ Juveniles II } \\
\hline Altura total $(\mathrm{cm})$ & 10 & $326.9 \pm 42.5(280-400)$ & 12 & $225.4 \pm 33.9(170-290)$ & ** \\
\hline Número de hojas & 10 & $5.6 \pm 1.1(4-7)$ & 12 & $5.7 \pm 1.5(3-7)$ & - \\
\hline Número de pinnas & 20 & $64 \pm 15.2(31+82)$ & 24 & $49.5 \pm 11.9(24-70)$ & ** \\
\hline Largo vaina-pecíolo (cm) & 10 & $142.2 \pm 48.5(79-220)$ & 12 & $106 \pm 22.3(60-130)$ & * \\
\hline Largo raquis $(\mathrm{cm})$ & 10 & $251.7 \pm 66.8(110-320)$ & 12 & $179.7 \pm 35.8(130-250)$ & ** \\
\hline Largo pinna distal (cm) & 10 & $44.4 \pm 9.8(30-65)$ & 12 & $42.2 \pm 7.9(34-60)$ & - \\
\hline Ancho pinna distal (cm) & 10 & $1.9 \pm 0.2(1.5-2)$ & 12 & $1.9 \pm 0.6(1-3)$ & - \\
\hline Largo pinna media $(\mathrm{cm})$ & 10 & $63.3 \pm 11.0(48-83)$ & 12 & $55.1 \pm 10.1(39-74)$ & - \\
\hline Ancho pinna media (cm) & 10 & $2.7 \pm 0.5(2-3)$ & 12 & $2.2 \pm 0.3(2-2.5)$ & * \\
\hline Largo pinna proximal (cm) & 10 & $45.9 \pm 9.1(34-64)$ & 12 & $45 \pm 10.9(22-66)$ & - \\
\hline Ancho pinna proximal $(\mathrm{cm})$ & 10 & $1.6 \pm 0.3(1-2)$ & 12 & $1.4 \pm 0.7(1-3)$ & - \\
\hline Largo aguijones vaina $(\mathrm{cm})$ & 50 & $4.6 \pm 2.0(0-8)$ & 60 & $3.4 \pm 2.4(0-8)$ & * \\
\hline \multicolumn{6}{|l|}{ Juveniles I } \\
\hline Altura total $(\mathrm{cm})$ & 15 & $156.2 \pm 37.4(90-210)$ & 18 & $132.2 \pm 40.9(68-190)$ & - \\
\hline Número de hojas & 15 & $4.9 \pm 1.3(3-7)$ & 18 & $5.7 \pm 1.3(4-8)$ & - \\
\hline Número de pinnas & 30 & $29.5 \pm 12.7(6-48)$ & 36 & $11.8 \pm 5.9(3-23)$ & ** \\
\hline Largo vaina-pecíolo (cm) & 15 & $95.3 \pm 29.7(30-157)$ & 18 & $65 \pm 17.8(41-110)$ & ** \\
\hline Largo raquis $(\mathrm{cm})$ & 15 & $123.1 \pm 40.3(39-183)$ & 18 & $78.2 \pm 18.3(44-112)$ & $* *$ \\
\hline Largo pinna distal (cm) & 15 & $50.5 \pm 7.0(39-64)$ & 18 & $52.3 \pm 9.4(36-70)$ & - \\
\hline Ancho pinna distal (cm) & 15 & $3.2 \pm 2.4(1.5-8)$ & 18 & $5.4 \pm 2.8(1-11)$ & * \\
\hline Largo pinna media (cm) & 15 & $48.8 \pm 8.7(37-65)$ & 18 & $44.9 \pm 7.5(29-60)$ & - \\
\hline Ancho pinna media (cm) & 15 & $2.4 \pm 0.9(1.5-5)$ & 18 & $2.4 \pm 1.6(1-5)$ & - \\
\hline Largo pinna proximal (cm) & 15 & $39.8 \pm 9.2(26-58)$ & 18 & $42.8 \pm 6.0(31-52)$ & - \\
\hline Ancho pinna proximal (cm) & 15 & $1.9 \pm 0.9(1-5)$ & 18 & $2.2 \pm 1.0(1-4)$ & - \\
\hline Largo aguijones vaina $(\mathrm{cm})$ & 75 & $3.2 \pm 1.9(0-8)$ & 90 & $1.5 \pm 2.2(0-8)$ & ** \\
\hline \multicolumn{6}{|l|}{ Plántulas } \\
\hline Número hojas & 37 & $3.9 \pm 1.5(1-7)$ & 59 & $5.3 \pm 2.0(1-9)$ & ** \\
\hline Largo pecíolo (cm) & 37 & $19.2 \pm 6.3(9-33)$ & 59 & $20.7 \pm 7.9(7-42)$ & - \\
\hline Largo raquis $(\mathrm{cm})$ & 37 & $14.8 \pm 3.1(9-21)$ & 59 & $18.5 \pm 5.8(6-29)$ & ** \\
\hline Ancho lámina (cm) & 37 & $4.6 \pm 1.4(2.8-9.5)$ & 59 & $7.1 \pm 2.9(1.5-14)$ & $* *$ \\
\hline
\end{tabular}

pinnas, largo de la vaina-pecíolo, largo del raquis, ancho de la pinna media y largo de los aguijones. (3) Cinco caracteres son significativamente diferentes en juveniles I: número de pinnas, largo de la vaina-pecíolo, largo del raquis, ancho de la pinna distal y largo de los aguijones. (4) Tres caracteres son significativamente diferentes en plántulas: número de hojas, largo del raquis y ancho de la lámina.

Variabilidad vegetativa entre Astrocaryum chonta y $A$. javarense (Tabla 3).- (1) Entre adultos de las dos especies, 13 caracteres son significativamente diferentes: altura total, largo del tronco, diámetro del tronco, número de hojas, número de pinnas, largo del raquis, largo de la pinna distal, ancho de la pinna distal, largo de la pinna media, ancho de la pinna media, largo de la pinna proximal, ancho de pinna proximal y largo de los aguijones. (2) Entre juveniles II de las dos especies, siete caracteres son significativamente diferentes: número de hojas, largo del raquis, ancho de la pinna distal, largo de la pinna media, ancho de la pinna media, largo de la pinna proximal y ancho de la pinna proximal. (3) Entre juveniles I de las dos especies, seis caracteres son significativamente diferentes: número de pinnas, ancho de la pinna distal, largo de la pinna media, largo de la pinna proximal, ancho de la pinna proximal y largo de los aguijones. (4) Las plántulas de las dos especies solo difieren por el número de hojas. 


\section{Discusión}

Al comparar Astrocaryum gynacanthum y Astrocaryum minus, Trail (1877) observó que son diferentes, pero que estas diferencias difícilmente se pueden expresar en una descripción. Las consideraciones de Trail se aplican a los caracteres vegetativos y también valen para las especies de la sección Huicungo, que parecen ser muy uniformes (Kahn \& Millán 2013). Sin embargo, el presente trabajo encontramos diferencias significativas en caracteres vegetativos a nivel intra e interespecíficos en Astrocaryum chonta y A. javarense.

La variabilidad vegetativa intrapoblacional resulta ser mayor en Astrocaryum javarense (número de caracteres significativa- mente diferentes: 10 de 14 en adultos, 6 de 12 en juveniles II, 5 de 12 en juveniles I y 3 de 4 en plántulas) que en Astrocaryum chonta (6 de 14 en adultos, 3 de 12 en juveniles II, 3 de 12 en juveniles I, 2 de 4 en plántulas). La variabilidad vegetativa intrapoblacional de Astrocaryum javarense es similar a la encontrada en $A$. perangustatum por Aponte et al. (2011). Las dos especies estudiadas muestran variabilidad vegetativa poblacional tal como fue reportado en otras especies de palmeras como: Euterpe precatoria (Kahn \& Granville 1992), Cocos nucifera (ZizumboVillareal \& Piñero 1998), Geonoma cuneata (Borchsenius 1999).

A pesar de presentar alta similitud fisonómica, con tronco corto a mediano y hojas largas, Astrocaryum chonta y A. javarense

Tabla 3. Variabilidad vegetativa entre Astrocaryum chonta y $A$. javarense: adultos, juveniles II, juveniles I y plántulas ( ${ }^{* *} p<0.01$, $\left.{ }^{*} p<0.05,-p>0.05\right)$.

\begin{tabular}{|c|c|c|c|c|c|}
\hline \multirow[b]{2}{*}{ Adultos } & \multicolumn{2}{|r|}{ Astrocaryum chonta } & \multicolumn{2}{|r|}{ Astrocaryum javarense } & \multirow[b]{2}{*}{$\mathbf{p}$} \\
\hline & $\mathbf{N}$ & Media \pm DS (min-max) & $\mathbf{N}$ & Media \pm DS (min-max) & \\
\hline Altura total $(\mathrm{cm})$ & 40 & $948.3 \pm 201.6(700-1420)$ & 40 & $513.9 \pm 90.3(350-890)$ & ** \\
\hline Largo tronco $(\mathrm{cm})$ & 40 & $383.7 \pm 198.4(50-700)$ & 40 & $69.9 \pm 50.7(50-300)$ & ** \\
\hline Diámetro tronco $(\mathrm{cm})$ & 40 & $17.4 \pm 5.0(9-27.7)$ & 40 & $11.6 \pm 6.4(10-50)$ & ** \\
\hline Número de hojas & 40 & $10.7 \pm 2.2(7-15)$ & 40 & $8.0 \pm 1.9(5-13)$ & ** \\
\hline Número de pinnas & 240 & $103.2 \pm 11.3(74-130)$ & 240 & $94.2 \pm 14.5(65-130)$ & ** \\
\hline Largo vaina-pecíolo (cm) & 120 & $189.2 \pm 28.5(120-282)$ & 120 & $198.1 \pm 45.8(120-360)$ & - \\
\hline Largo del raquis $(\mathrm{cm})$ & 120 & $527.9 \pm 83.8(350-700)$ & 120 & $438.2 \pm 84.0(220-623)$ & ** \\
\hline Largo pinna distal (cm) & 120 & $51 \pm 13.1(29-89)$ & 120 & $44.8 \pm 11.4(20-80)$ & ** \\
\hline Ancho pinna distal $(\mathrm{cm})$ & 120 & $2.5 \pm 0.7(1.3-5)$ & 120 & $2.0 \pm 0.5(1-3.5)$ & ** \\
\hline Largo pinna media $(\mathrm{cm})$ & 120 & $117.9 \pm 17.2(88-160)$ & 120 & $89.8 \pm 16.3(60-135)$ & ** \\
\hline Ancho pinna media (cm) & 120 & $6.0 \pm 1.1(4-9)$ & 120 & $4.0 \pm 0.9(1-7)$ & ** \\
\hline Largo pinna proximal $(\mathrm{cm})$ & 120 & $79.5 \pm 22.1(38-132)$ & 120 & $60.3 \pm 15.2(22-105)$ & ** \\
\hline Ancho pinna proximal $(\mathrm{cm})$ & 120 & $2.7 \pm 1.0(1-7.2)$ & 120 & $1.9 \pm 0.6(1-3.5)$ & ** \\
\hline Largo aguijones vaina $(\mathrm{cm})$ & 200 & $16.0 \pm 5.5(5-33.6)$ & 200 & $13.3 \pm 9.4(2.5-45)$ & ** \\
\hline \multicolumn{6}{|l|}{ Juveniles II } \\
\hline Altura total $(\mathrm{cm})$ & 18 & $324.7 \pm 107.6(200-600)$ & 22 & $271.5 \pm 63.7(170-400)$ & - \\
\hline Número de hojas & 18 & $7.2 \pm 1.8(4-11)$ & 22 & $5.6 \pm 1.3(3-7)$ & ** \\
\hline Número de pinnas & 36 & $56.3 \pm 20.4(27-89)$ & 44 & $56.1 \pm 15.2(24-82)$ & - \\
\hline Largo vaina-pecíolo (cm) & 18 & $146.3 \pm 52.4(92-290)$ & 22 & $122.5 \pm 40.1(60-220)$ & - \\
\hline Largo raquis $(\mathrm{cm})$ & 18 & $266.6 \pm 69.4(150-380)$ & 22 & $212.4 \pm 62.7(110-320)$ & * \\
\hline Largo pinna distal $(\mathrm{cm})$ & 18 & $42.0 \pm 13.2(19-75)$ & 22 & $43.2 \pm 8.7(30-65)$ & - \\
\hline Ancho pinna distal $(\mathrm{cm})$ & 18 & $2.6 \pm 1.0(1-5.5)$ & 22 & $1.9 \pm 0.5(1-3)$ & ** \\
\hline Largo pinna media $(\mathrm{cm})$ & 18 & $74.4 \pm 19.3(49-105)$ & 22 & $58.8 \pm 11.1(39-83)$ & ** \\
\hline Ancho pinna media $(\mathrm{cm})$ & 18 & $3.3 \pm 0.9(2-5)$ & 22 & $2.4 \pm 0.4(2-3)$ & ** \\
\hline Largo pinna proximal (cm) & 18 & $68.4 \pm 33.6(8-134)$ & 22 & $45.4 \pm 9.9(22-66)$ & $* *$ \\
\hline Ancho pinna proximal $(\mathrm{cm})$ & 18 & $2.9 \pm 1.9(1-7)$ & 22 & $1.5 \pm 0.5(1-3)$ & ** \\
\hline Largo aguijones vaina $(\mathrm{cm})$ & 90 & $4.6 \pm 2.6(0-12)$ & 110 & $4.0 \pm 2.3(0-8)$ & - \\
\hline \multicolumn{6}{|l|}{ Juveniles I } \\
\hline Altura total $(\mathrm{cm})$ & 37 & $160 \pm 71.3(70-400)$ & 33 & $143.1 \pm 40.6(68-210)$ & - \\
\hline Número de hojas & 37 & $6.0 \pm 1.5(4-9)$ & 33 & $5.4 \pm 1.3(3-8)$ & - \\
\hline Número de pinnas & 74 & $10.3 \pm 6.5(3-37)$ & 66 & $19.9 \pm 13.0(3-48)$ & ** \\
\hline Largo vaina-pecíolo (cm) & 37 & $90.2 \pm 29.5(34-152)$ & 33 & $78.8 \pm 28.1(30-157)$ & - \\
\hline Largo raquis $(\mathrm{cm})$ & 37 & $97.3 \pm 42.1(26-212)$ & 33 & $98.6 \pm 37.5(39-183)$ & - \\
\hline Largo pinna distal (cm) & 37 & $54.8 \pm 14.6(14-80)$ & 33 & $51.5 \pm 8.3(36-70)$ & - \\
\hline Ancho pinna distal $(\mathrm{cm})$ & 37 & $6.3 \pm 4.0(1.5-14)$ & 33 & $4.4 \pm 2.8(1-11)$ & * \\
\hline Largo pinna media $(\mathrm{cm})$ & 37 & $57.5 \pm 12.0(33-78)$ & 33 & $46.7 \pm 8.2(29-65)$ & ** \\
\hline Ancho pinna media $(\mathrm{cm})$ & 37 & $2.7 \pm 1.9(1.5-11)$ & 33 & $2.4 \pm 1.3(1-5)$ & - \\
\hline Largo pinna proximal (cm) & 37 & $60.7 \pm 13.1(35-85)$ & 33 & $41.4 \pm 7.7(26-58)$ & ** \\
\hline Ancho pinna proximal $(\mathrm{cm})$ & 37 & $3.7 \pm 2.5(1-13)$ & 33 & $2.0 \pm 1.0(1-5)$ & ** \\
\hline Largo aguijones vaina $(\mathrm{cm})$ & 190 & $3.5 \pm 3.3(0-19)$ & 165 & $2.3 \pm 2.2(0-8)$ & ** \\
\hline \multicolumn{6}{|l|}{ Plántulas } \\
\hline Número de hojas & 112 & $3.8 \pm 1.4(1-7)$ & 96 & $4.7 \pm 1.9(1-9)$ & ** \\
\hline Largo pecíolo (cm) & 112 & $18.9 \pm 7.5(7-54)$ & 96 & $20.2 \pm 7.3(7-42)$ & - \\
\hline Largo raquis $(\mathrm{cm})$ & 112 & $17.3 \pm 5.5(7-34)$ & 96 & $17.1 \pm 5.2(6-29)$ & - \\
\hline Ancho lamina $(\mathrm{cm})$ & 112 & $6.4 \pm 2.4(2-13)$ & 96 & $6.2 \pm 2.7(1.5-14)$ & - \\
\hline
\end{tabular}


muestran diferencias significativas en los datos biométricos: 13/14 en adultos, 7/12 en juveniles II, 6/12 en juveniles I y 1/4 en plántulas. Los valores promedios de los caracteres significativos son mayores en Astrocaryum chonta que en $A$. javarense, a excepción del carácter longitud de la vaina-pecíolo, que es mayor en Astrocaryum javarense. Astrocaryum chonta usualmente presenta palmeras que alcanzan los niveles superiores del sotobosque (10 m de altura); mientras Astrocaryum javarense raramente pasa los niveles intermedios del sotobosque (5-7 $\mathrm{m}$ de altura). Similar patrón de diferencias morfológicas pero no cuantificadas encontraron Kahn y Granville (1998) entre Astrocaryum gynacanthum y $A$. minus, estas dos especies pertenecen a la misma sección (Munbaca) y ambas presentan la misma forma de crecimiento palmeras pequeñas delgadas (Kahn 2008, Balslev et al. 2011).

La similitud fisonómica entre las especies esconde las diferencias morfológicas, estas últimas salen claramente del análisis biométrico. Pero, es evidente que no se puede hacer un análisis morfométrico para identificar las especies en el campo. Será la combinación de varios caracteres, algunos vegetativos con otros de flores y frutos los que permitirían identificar la especie sin ninguna ambigüedad (Kahn \& Millán 2013). Sin embargo, los resultados ponen en evidencia algunos indicadores útiles, como el tamaño de las palmeras, principalmente la altura alcanzada por el tronco de Astrocaryum chonta y la longitud de sus hojas que permiten diferenciarla de $A$. javarense, siendo esta última una palmera más pequeña. En el área estudiada las poblaciones de las dos especies están contiguas, pero claramente ubicadas en dos formaciones geomorfológicas distintas. El criterio ecológico/ geomorfológico permite también corroborar la identificación de las especies: Astrocaryum chonta se encuentra sobre los depósitos aluviales ubicados en las terrazas más bajas, mientras $A$. javarense crece sobre los suelos arcillosos en las terrazas más altas de la zona interfluvial.

\section{Agradecimientos}

Agradecemos al Centro de Investigaciones Jenaro Herrera (CIHJ-IIAP) y al señor Italo Meléndez por su colaboración y guía en el trabajo de campo.

\section{Literatura citada}

Anthelme F., R. Montúfar-Galárraga \& J.C. Pintaud. 2010. Caracterización de la resilencia ecológica de poblaciones de palmeras. Ecología en Bolivia 45(3): 23-29.

Aponte H., F. Kahn \& B. Millán. 2011. Vegetative adaptability of the Peruvian palm Astrocaryum perangustatum to deforestation. Revista peruana de Biologia 18 (2):179-183.
Balslev H., F. Kahn, B. Millán, J. Svenning, et al. 2011. Species Diversity and Growth Forms in Tropical American Palm Communities. The Botanical Review 77:381-425.

Borchsenius F. 1999. Morphological variation in Geonoma cuneata in Western Ecuador. Memoirs of The New York Botanical Garden 83: 131-139.

Escobedo R., L. Bendayán, C. Rojas, F. Rodríguez \& L. Marquina. 1994. Estudio detallado de suelos de la zona "Fernando Lores"-Tamshiyacu (Región Loreto). Documento Técnico No 05. IIAP. Iquitos-Perú.

García J.L. 1975. Caracterización del estado nutricional de los suelos de la Colonización Jenaro Herrera I mediante la técnica del elemento faltante. Tesis para optar el Título de Ingeniero Agrónomo. Universidad Nacional de la Amazonía Peruana. Iquitos-Perú.

Hoag R. E. 1987. Characterization of soils on floodplains of tributaries flowing into the Amazon River in Peru. North Carolina State University. 330 pp.

Kahn F. 2008. El género Astrocaryum (Arecaceae). Revista peruana de Biologia 15 (supl. 1): 031- 048.

Kahn F. \& J.-J. de Granville 1992. Palms in forest ecosystems of Amazonia. Springer Verlag, Berlin, Heidelberg, New York, Tokyo. $226 \mathrm{pp}$.

Kahn F. \& J.J. de Granville. 1998. Astrocaryum minus, Rediscovered in French Guiana. Principes 42(3): 171-178.

Kahn F. \& B. Millán. 2013. Los Palmeras Huicungo-The Huicungo palms. UNMSM-IRD, Lima, $173 \mathrm{p}$.

Kahn F., B. Millán, J.C. Pintaud \& M. Machahua. 2011. Detailed assessment of the distribution of Astrocaryum sect. Huicungo (Arecaceae) in Peru. Revista peruana de Biologia 18 (3):279-282.

López Parodi J. \& D. Freitas. 1990. Geographical aspects of forested wetlands in the Lower Ucayali, Peruvian Amazonia. Forest Ecology and Management 33/34:157-168.

Lorenzi H., L.R. Noblick, F. Kahn \& E. Ferreira. 2010. Flora BrasileiraArecaceae. Instituto Plantarum, Nova Odessa, 384 pp.

Rodríguez F. 1990. Los suelos de áreas inundables de la Amazonía Peruana: Potencial, limitaciones y estrategias para su investigación. Folia Amazónica IIAP 2: 7-25.

SPSS Inc. 2008. SPSS Statistics for Windows, Version 17.0. Chicago.

Trail J.W.H. 1877. Description of new species and varieties of palms collected in the valley of the Amazon in north Brazil in 1874. Journal of Botany 15:75-81.

Vormisto J., J-C. Svenning, P. Hall \& H. Balslev. 2004. Diversity and dominance in palms (Arecaceae) communities in tierra firme forest in the western Amazon basin. Journal of Ecology 92:577-588.

Zizumbo-Villarreal D. \& D. Piñero. 1998. Pattern of morphological variation and diversity of Cocos nucifera (Arecaceae) in Mexico. American Journal of Botany 85(6):855-865. 